\title{
Aqueous phase synthesis of polysubstituted pyrimidines/pyrrolidines catalyzed by $\beta$-cyclodextrin
}

\author{
Jilla Shankar, Gaddam Satish, Katla Ramesh and Nageswar Yadavalli Venkata Durga * \\ Council of Scientific and Industrial Research-Medicinal Chemistry and Pharmacology Division, Indian Institute of Chemical Technology, Uppal Road, \\ Hyderabad 500-007, India
}

*Corresponding author at: Council of Scientific and Industrial Research-Medicinal Chemistry and Pharmacology Division, Indian Institute of Chemical Technology, Uppal Road, Hyderabad 500-007, India.

Tel.: +91.040.27191654. Fax: +91.040.27193189. E-mail address: dryvdnageswar@gmail.com (N.Y.V.Durga).

\section{COMMUNICATION INFORMATION}

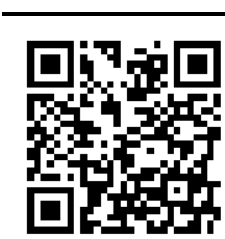

DOI: 10.5155 /eurjchem.5.3.541-544.1049

Received: 13 March 2014

Received in revised form: 16 April 2014

Accepted: 22 April 2014

Online: 30 September 2014

\section{KEYWORDS}

Amines

Formaldehyde

$\beta$-Cyclodextrin

Pyrimidine derivatives

Pyrrolidine derivatives

Dimethyl/diethyl acetylene dicarboxylates

\section{Introduction}

Pyrimidine derivatives are versatile building blocks in synthetic organic chemistry and many bioactive molecules [14] with pyrimidine skeleton served as M1 muscarinic receptor agonists for the treatment of Alzheimer's disease [5,6] and human immune deficiency virus (HIV) protease inhibitors [7]. Pyrimidine moiety is an important class of $\mathrm{N}$-containing heterocyclic system $[8,9]$, which exhibits wide spectrum of biological activities such as bactericidal [10], fungicidal [11], analgesic [12], anti-hypertensive [13], antimicrobial [14] and anti-infective properties [15]. Research in potential polysubstituted pyrimidines prompted for the development of a variety of synthetic strategies. Vishwakarma et. al. reported the synthesis of poly-substituted 1,2,3,4-tetrahydropyrimidines [16]. Das et. al. reported the synthesis of poly substituted pyrrolidines and tetra-hydropyrimidines involving indium as a catalyst in aqueous medium [17]. Zhu et. al. described a one-pot synthesis of poly-substituted tetrahydropyrimidines via the proton-promoted MCRs [18].

In view of different biological activities associated with pyrimidine/pyrrolidine derivatives and in continuation of our interest in the use of cyclodextrins as mild and efficient biomimetic catalysts in promoting various organic transformations [19-27], we here in report the synthesis of poly-substituted tetra-hydropyrimidine/pyrrolidine derivatives by the reaction of substituted anilines with but-2ynedioates and formaldehyde under neutral conditions involving $\beta$-cyclodextrin in water medium.

\section{Experimental}

\subsection{Instrumentation}

All chemicals were purchased from Fluka and S. D. Fine Chemicals and directly used for the synthesis. All reactions were carried out without any special precautions in an atmosphere of air. Analytical Thin Layer Chromatography (TLC) was carried out by using silica gel $60 \mathrm{~F}_{254}$ pre-coated plates. Visualization was accomplished with UV lamp or $\mathrm{I}_{2}$ staining. Melting point was obtained by Fischer-Johns meltingpoint apparatus and uncorrected. All products were characterized by their NMR and Mass spectra. ${ }^{1} \mathrm{H}$ NMR were recorded on $200 \mathrm{MHz}$, in $\mathrm{CDCl}_{3}$ using TMS as the internal standard and chemical shifts were reported in parts per million $(\mathrm{ppm}, \delta$ ) downfield from the tetramethylsilane. NMR Spectra: Varian 200 spectrometer; in $\mathrm{CDCl}_{3} ; \delta$ in ppm, $J$ in $\mathrm{Hz}$. Mass spectra: $V G$ Autospec; in $m / z$. IR were recorded on a Thermo Nicolet Nexus 670 FT-IR spectrometer. 
Table 1. $\beta$-CD catalysed synthesis of polysubstituted pyrrolidines a.

\begin{tabular}{llllll}
\hline Entry & $\mathbf{R}$ & $\mathbf{R}^{\mathbf{1}}$ & Product & Time (h) & Yield (\%) \\
\hline 1 & $4-\mathrm{CF}_{3}-\mathrm{C}_{6} \mathrm{H}_{4}$ & $4 \mathrm{a}$ & 4.5 & 75 \\
2 & $4-\mathrm{CH}_{6} \mathrm{C}_{6} \mathrm{H}_{4}$ & $\mathrm{CH}_{3}$ & $4 \mathrm{~b}$ & 4.5 & 75 \\
3 & $4-\mathrm{CH}_{3}-\mathrm{C}_{6} \mathrm{H}_{4}$ & $\mathrm{CH}_{3}$ & $4 \mathrm{c}$ & 4.5 & 70 \\
4 & $\mathrm{C}_{6} \mathrm{H}_{5}$ & $\mathrm{CH}_{3}$ & $4 \mathrm{~d}$ & 4.5 & 75 \\
5 & $3-\mathrm{CH}_{3}-\mathrm{C}_{6} \mathrm{H}_{4}$ & $\mathrm{CH}_{2}-\mathrm{CH}_{3}$ & $4 \mathrm{e}$ & 4.5 & 68 \\
\hline
\end{tabular}

a Reaction conditions: Amine (1.0 mmol), DMAD/DEAD (1.0 mmol), Formaldehyde (4.0 mmol), $\beta$-Cyclodextrin $(10 \mathrm{~mol} \%), 60{ }^{\circ} \mathrm{C}$.

b Isolated yield.

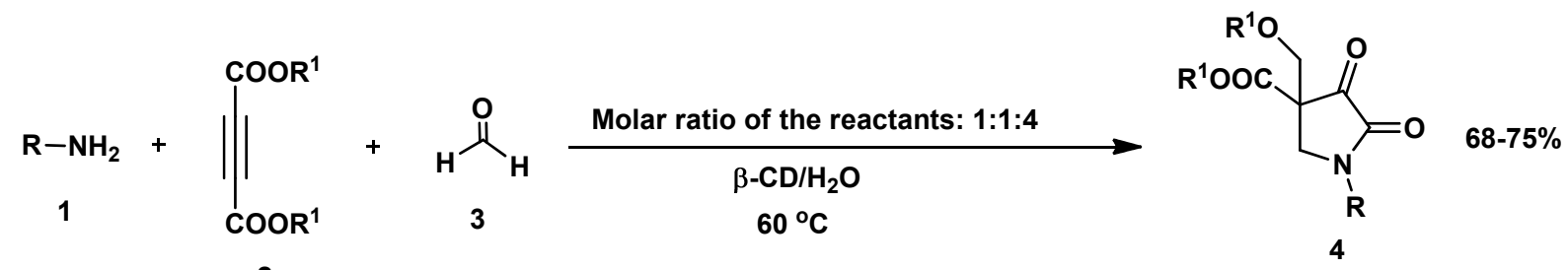

2

\author{
$\mathrm{R}=-\mathrm{C}_{6} \mathrm{H}_{5}, 4-\mathrm{CH}_{3} \mathrm{C}_{6} \mathrm{H}_{4}, 4-\mathrm{HOC}_{6} \mathrm{H}_{4}, 4-\mathrm{CF}_{3} \mathrm{C}_{6} \mathrm{H}_{4}, 3-\mathrm{CH}_{3} \mathrm{C}_{6} \mathrm{H}_{4}$$$
\mathrm{R}^{1}=-\mathrm{CH}_{3},-\mathrm{C}_{2} \mathrm{H}_{5}
$$

Scheme 1

\subsection{Synthesis}

\subsubsection{General procedure for the synthesis of poly- substituted pyrrolidines}

$\beta$-Cyclodextrin (1.135 g, $1 \mathrm{mmol}$ ) was dissolved in water $\left(15 \mathrm{~mL}\right.$ ) by warming up to $60{ }^{\circ} \mathrm{C}$ until a clear solution was formed. To this clear solution, aniline $(1.0 \mathrm{mmol})$ was added and stirred for $10 \mathrm{~min}$, and then dimethylacetylene dicarboxylate $(1.0 \mathrm{mmol})$ was added. After stirring for half an hour 4 equivalents of formaldehyde $(4.0 \mathrm{mmol})$ was added. The reaction mixture was stirred until completion of the reaction as indicated by TLC. The reaction mixture was cooled and $\beta$ Cyclodextrin was filtered. The aqueous phase was extracted with ethyl acetate $(3 \times 10 \mathrm{~mL})$ and the organic layers were washed with water, saturated brine solution, and dried over anhydrous $\mathrm{Na}_{2} \mathrm{SO}_{4}$. The combined organic layers were evaporated under reduced pressure and the resulting crude product was purified by column chromatography by using ethyl acetate: hexane $(2: 8, v: v)$ as eluent to give the corresponding ethyl 3-(ethoxymethyl)-4,5-dioxo-1-phenylpyrrolidine-3carboxylate as pure product in good yield (Scheme 1).

Methyl 1-(4-trifluoromethylphenyl)-3(methoxy-methyl)-4,5dioxopyrrolidine-3 carboxylate (Table 1, Entry 1): Yield: 75\%. Color: Yellow oil. ${ }^{1} \mathrm{H}$ NMR $\left(200 \mathrm{MHz}, \mathrm{CDCl}_{3}, \delta, \mathrm{ppm}\right): 8.05$ (d, $2 \mathrm{H}$, $J=8.0 \mathrm{~Hz}, \mathrm{Ar}-\mathrm{H}), 7.74(\mathrm{~d}, 2 \mathrm{H}, J=8.0 \mathrm{~Hz}, \mathrm{Ar}-\mathrm{H}), 4.55(\mathrm{~d}, 1 \mathrm{H}, J=$ $\left.12.0 \mathrm{~Hz}, \mathrm{CH}_{2}\right), 4.24\left(\mathrm{~d}, 1 \mathrm{H}, J=12.0 \mathrm{~Hz}, \mathrm{CH}_{2}\right), 3.97(\mathrm{~d}, 1 \mathrm{H}, J=10.0$ $\mathrm{Hz}, \mathrm{CH}_{2}$ ), 3.87 (d, $\left.1 \mathrm{H}, J=10.0 \mathrm{~Hz}, \mathrm{CH}_{2}\right), 3.81\left(\mathrm{~s}, 3 \mathrm{H}, \mathrm{OCH}_{3}\right), 3.36$ $\left(\mathrm{s}, 3 \mathrm{H}, \mathrm{OCH}_{3}\right)$. FT-IR $\left(\mathrm{KBr}, \mathrm{v}, \mathrm{cm}^{-1}\right): 1778,1740,1615,1462$. MS (ESI, $m / z): 346[\mathrm{M}+\mathrm{H}]^{+}$.

Methyl 1-(4-hydroxyphenyl)-3(methoxymethyl)-4,5-dioxo pyrrolidine-3-carboxylate (Table 1, Entry 2): Yield: 75\%. Color: Yellow oil. ${ }^{1} \mathrm{H}$ NMR $\left(200 \mathrm{MHz}, \mathrm{CDCl}_{3}, \delta, \mathrm{ppm}\right): 7.67(\mathrm{~d}, 2 \mathrm{H}, J=8.0$ $\mathrm{Hz}, \mathrm{Ar}-\mathrm{H}), 6.89(\mathrm{~d}, 2 \mathrm{H}, J=8.0 \mathrm{~Hz}, \mathrm{Ar}-\mathrm{H}), 4.49(\mathrm{~d}, 1 \mathrm{H}, J=12.0 \mathrm{~Hz}$, $\left.\mathrm{CH}_{2}\right), 4.25\left(\mathrm{~d}, 1 \mathrm{H}, J=12.0 \mathrm{~Hz}, \mathrm{CH}_{2}\right), 4.00\left(\mathrm{~d}, 1 \mathrm{H}, J=10.0 \mathrm{~Hz}, \mathrm{CH}_{2}\right)$, $3.92\left(\mathrm{~d}, 1 \mathrm{H}, J=10.0 \mathrm{~Hz}, \mathrm{CH}_{2}\right), 3.85\left(\mathrm{~s}, 3 \mathrm{H}, \mathrm{OCH}_{3}\right), 3.36(\mathrm{~s}, 3 \mathrm{H}$, $\left.\mathrm{OCH}_{3}\right), 2.86(\mathrm{~s}, 1 \mathrm{H}, \mathrm{OH})$. FT-IR $\left(\mathrm{KBr}, \mathrm{v}, \mathrm{cm}^{-1}\right): 3378,1775$ 1695, 1517, 1462. MS (ESI, $m / z): 294[\mathrm{M}+\mathrm{H}]^{+}$.

Methyl 3-(methoxymethyl)-4, 5-dioxo-1-(p-tolyl) pyrrolidine3-carboxylate (Table 1, Entry 3): Yield: 70\%. Color: Yellow oil. ${ }^{1} \mathrm{H}$ NMR $\left(200 \mathrm{MHz}, \mathrm{CDCl}_{3}, \delta, \mathrm{ppm}\right): 7.75(\mathrm{~s}, 1 \mathrm{H}, \mathrm{Ar}-\mathrm{H}), 7.66(\mathrm{~d}$, $1 \mathrm{H}, J=8.0 \mathrm{~Hz}, \mathrm{Ar}-\mathrm{H}), 7.30(\mathrm{t}, 1 \mathrm{H}, J=8.0 \mathrm{~Hz}, \mathrm{Ar}-\mathrm{H}), 7.12(\mathrm{~d}, 1 \mathrm{H}$, $J=8.0 \mathrm{~Hz}, \mathrm{Ar}-\mathrm{H}), 4.55\left(\mathrm{~d}, 1 \mathrm{H}, J=12.0 \mathrm{~Hz}, \mathrm{CH}_{2}\right), 4.26(\mathrm{~d}, 1 \mathrm{H}, J=$ $\left.12.0 \mathrm{~Hz}, \mathrm{CH}_{2}\right), 3.96\left(\mathrm{~d}, 1 \mathrm{H}, J=10.0 \mathrm{~Hz}, \mathrm{CH}_{2}\right), 3.85(\mathrm{~d}, 1 \mathrm{H}, J=10.0$
$\left.\mathrm{Hz}, \mathrm{CH}_{2}\right), 3.78$ (s, 3H, $\left.\mathrm{OCH}_{3}\right), 3.32\left(\mathrm{~s}, 3 \mathrm{H}, \mathrm{OCH}_{3}\right), 2.46(\mathrm{~s}, 3 \mathrm{H}$, $\mathrm{CH}_{3}$ ). FT-IR (KBr, $\left.v, \mathrm{~cm}^{-1}\right): 1776,1714,1519,1265 . \mathrm{MS}$ (ESI, $m / z): 292[\mathrm{M}+\mathrm{H}]+$.

Ethyl 3-(ethoxymethyl)-4,5-dioxo-1-phenylpyrrolidine-3carboxylate (Table 1, Entry 4): Yield: $75 \%$. Color: Yellow oil. ${ }^{1} \mathrm{H}$ NMR $\left(200 \mathrm{MHz}, \mathrm{CDCl}_{3}, \delta, \mathrm{ppm}\right): 7.88(\mathrm{~d}, 2 \mathrm{H}, J=8.0, \mathrm{Ar}-\mathrm{H}), 7.44$ $(\mathrm{m}, 2 \mathrm{H}, J=8.0 \mathrm{~Hz}, \mathrm{Ar}-\mathrm{H}), 7.28(\mathrm{~m}, 1 \mathrm{H}, J=12.0 \mathrm{~Hz}, \mathrm{Ar}-\mathrm{H}), 4.25$ $\left(\mathrm{m}, 3 \mathrm{H}, J=12.0 \mathrm{~Hz}, \mathrm{CH}_{2}\right), 4.23\left(\mathrm{~d}, 1 \mathrm{H}, J=12.0 \mathrm{~Hz}, \mathrm{CH}_{2}\right), 3.93(\mathrm{q}$ $\left.2 \mathrm{H}, J=10.0 \mathrm{~Hz}, \mathrm{OCH}_{2}\right), 3.56\left(\mathrm{q}, 2 \mathrm{H}, J=7.0 \mathrm{~Hz}, \mathrm{OCH}_{2}\right), 1.27(\mathrm{t}, 3 \mathrm{H}$, $\left.J=7.0 \mathrm{~Hz}, \mathrm{CH}_{3}\right), 1.07\left(\mathrm{t}, 3 \mathrm{H}, J=7.0 \mathrm{~Hz}, \mathrm{CH}_{3}\right)$. FT-IR $\left(\mathrm{KBr}, v, \mathrm{~cm}^{-1}\right)$ : 1778, 1735, 1598, 1485. MS (ESI, $m / z): 306[\mathrm{M}+\mathrm{H}]{ }^{+}$.

Ethyl 1-(3-methylphenyl)-3(ethoxymethyl)-4,5-dioxopyrroli dine-3-carboxylate (Table 1, Entry 5): Yield: 68\%. Color: Yellow oil. ${ }^{1} \mathrm{H}$ NMR $\left(200 \mathrm{MHz}, \mathrm{CDCl}_{3}, \delta\right.$, ppm): $7.82(\mathrm{~s}, 1 \mathrm{H}, \mathrm{Ar}-\mathrm{H}), 7.79$ (d, $1 \mathrm{H}, J=8.0, \mathrm{Ar}-\mathrm{H}), 7.44(\mathrm{t}, 1 \mathrm{H}, J=8.0 \mathrm{~Hz}, \mathrm{Ar}-\mathrm{H}), 7.19$ (d, $1 \mathrm{H}, J$ = 8.0, aro ), $4.50\left(1 \mathrm{H}, \mathrm{d}, \mathrm{J}=12.0 \mathrm{~Hz}, \mathrm{CH}_{2}\right), 4.28\left(3 \mathrm{H}, \mathrm{m}, \mathrm{CH}_{2}\right), 3.97$ $\left(2 \mathrm{H}, \mathrm{q}, \mathrm{J}=7.0 \mathrm{~Hz}, \mathrm{CH}_{2}\right), 3.49\left(2 \mathrm{H}, \mathrm{q}, \mathrm{J}=7.0 \mathrm{~Hz}, \mathrm{CH}_{2}\right), 2.45(3 \mathrm{H}, \mathrm{s}$, $\left.\mathrm{CH}_{3}\right), 1.29\left(\mathrm{t}, 3 \mathrm{H}, J=7.0 \mathrm{~Hz}, \mathrm{CH}_{3}\right), 1.11\left(\mathrm{t}, 3 \mathrm{H}, J=7.0 \mathrm{~Hz}, \mathrm{CH}_{3}\right)$. FT-IR (KBr, v, $\left.\mathrm{cm}^{-1}\right)$ : 1772, 1710, 1517, 1255. MS (ESI, $\mathrm{m} / \mathrm{z}$ ): $320[\mathrm{M}+\mathrm{H}]^{+}$.

\subsubsection{General procedure for the synthesis of 1,2,3,6-tetra hydropyrimidines}

$\beta$-Cyclodextrin (1.135 g, $1 \mathrm{mmol}$ ) was dissolved in water $(15 \mathrm{~mL})$ by warming up to $60{ }^{\circ} \mathrm{C}$ until a clear solution was formed. To this clear solution, aniline $(1.0 \mathrm{mmol})$ was added and stirred for $10 \mathrm{~min}$, and then dimethylacetylene dicarboxylate $(1.0 \mathrm{mmol})$ was added. After stirring for half an hour another 1 equivalent of aniline $(1.0 \mathrm{mmol})$ was added followed by the addition of 4 equivalents of formaldehyde (4.0 mmol). The reaction mixture was stirred until completion of the reaction as indicated by TLC. The reaction mixture was cooled and $\beta$-CD was filtered. The aqueous phase was extracted with ethyl acetate $(3 \times 10 \mathrm{~mL})$ and the organic layers were washed with water, saturated brine solution, and dried over anhydrous $\mathrm{Na}_{2} \mathrm{SO}_{4}$. The combined organic layers were evaporated under reduced pressure and the resulting crude product was purified by column chromatography by using ethyl acetate: hexane $(2: 8, v: v)$ as eluent to give the corresponding dimethyl 1,3-diphenyl-1,2,3,6-tetrahydropyrimidine-4,5-dicarboxylate as pure product in good yield (Scheme 2 ).

Dimethyl 1,3-diphenyl-1,2,3,6-tetrahydropyrimidine-4,5dicarboxylate (Table 2, Entry 1): Yield: 65\%. Color: Yellow oil. 
Table 2. $\beta$-CD catalysed synthesis of polysubstituted tetrahydropyrimidines a.

\begin{tabular}{llllll}
\hline Entry & $\mathbf{R}$ & $\mathbf{R}^{\mathbf{1}}$ & Product & Time (h) & Yield (\%) b \\
\hline 1 & $\mathrm{C}_{6} \mathrm{H}_{5}$ & $\mathrm{CH}_{3}$ & $5 \mathrm{a}$ & 4.5 & 65 \\
2 & $4-\mathrm{CCH}_{3}-\mathrm{C}_{6} \mathrm{H}_{4}$ & $\mathrm{CH}_{3}$ & $5 \mathrm{~b}$ & 4.5 & 68 \\
3 & $4-\mathrm{CH}_{3}-\mathrm{C}_{6} \mathrm{H}_{4}$ & $\mathrm{CH}_{3}$ & $5 \mathrm{c}$ & 4.5 & 67 \\
4 & $4-\mathrm{CF}_{3}-\mathrm{C}_{6} \mathrm{H}_{4}$ & $\mathrm{CH}_{3}$ & $5 \mathrm{~d}$ & 4.5 & 70 \\
5 & $4-\mathrm{CF}_{3}-\mathrm{C}_{6} \mathrm{H}_{4}$ & $\mathrm{CH}_{2}-\mathrm{CH}_{3}$ & $5 \mathrm{e}$ & 4.5 & 70 \\
\hline
\end{tabular}

a Reaction conditions: Amine (2.0 mmol), DMAD/DEAD (1.0 mmol), Formaldehyde $(4.0 \mathrm{mmol}), \beta$-Cyclodextrin $(10 \mathrm{~mol} \%), 60{ }^{\circ} \mathrm{C}$. b Isolated yield.

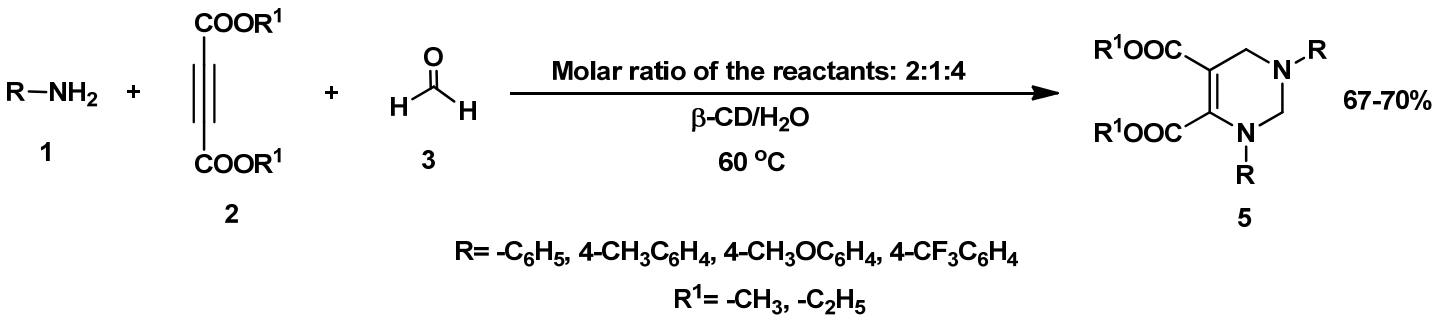

Scheme 2

${ }^{1} \mathrm{H}$ NMR (200 MHz, $\left.\mathrm{CDCl}_{3}, \delta, \mathrm{ppm}\right): 7.67(\mathrm{~d}, 2 \mathrm{H}, J=8.0 \mathrm{~Hz}$, Ar-H), 7.51 (d, 2H, $J=8.0 \mathrm{~Hz}, \mathrm{Ar}-\mathrm{H}), 7.44$ (d, $2 \mathrm{H}, J=8.0 \mathrm{~Hz}, \mathrm{Ar}-$ H), $6.84(\mathrm{~d}, 2 \mathrm{H}, J=8.0 \mathrm{~Hz}, \mathrm{Ar}-\mathrm{H}), 6.65(\mathrm{~d}, 2 \mathrm{H}, J=8.0 \mathrm{~Hz}, \mathrm{Ar}-\mathrm{H})$, $4.92\left(\mathrm{~s}, 2 \mathrm{H}, \mathrm{CH}_{2}\right), 4.35$ (s, 2H, $\left.\mathrm{CH}_{2}\right), 3.77\left(\mathrm{~s}, 3 \mathrm{H}, \mathrm{CH}_{3}\right), 3.65(\mathrm{~s}, 3 \mathrm{H}$, $\mathrm{CH}_{3}$ ). FT-IR (KBr, v, $\mathrm{cm}^{-1}$ ): 1749, 1715, 1617, 1336. MS (ESI, $m / z): 353[\mathrm{M}+\mathrm{H}]^{+}$.

Dimethyl 1,3-bis(4-methoxyphenyl)-1,2,3,6-tetrahydro pyrimidine-4,5-dicarboxylate (Table 2, Entry 2): Yield: 68\%. Color: Yellow oil. ${ }^{1} \mathrm{H}$ NMR $\left(200 \mathrm{MHz}^{\mathrm{CDCl}} 3, \delta, \mathrm{ppm}\right): 6.85(\mathrm{~d}, 2 \mathrm{H}$, $J=8.0 \mathrm{~Hz}, \mathrm{Ar}-\mathrm{H}), 6.77(\mathrm{~d}, 2 \mathrm{H}, J=8.0 \mathrm{~Hz}, \mathrm{Ar}-\mathrm{H}), 6.72(\mathrm{~d}, 2 \mathrm{H}, J=$ $8.0 \mathrm{~Hz}, \mathrm{Ar}-\mathrm{H}), 6.69$ (d, $2 \mathrm{H}, J=8.0 \mathrm{~Hz}, \mathrm{Ar}-\mathrm{H}), 4.74\left(\mathrm{~s}, 2 \mathrm{H}, \mathrm{CH}_{2}\right)$, $4.12\left(\mathrm{~s}, 2 \mathrm{H}, \mathrm{CH}_{2}\right), 3.74\left(\mathrm{~s}, 6 \mathrm{H}, \mathrm{OCH}_{3}\right), 3.69\left(\mathrm{~s}, 3 \mathrm{H}, \mathrm{CH}_{3}\right), 3.56(\mathrm{~s}$, $\left.3 \mathrm{H}, \mathrm{CH}_{3}\right)$. FT-IR (KBr, v, $\left.\mathrm{cm}^{-1}\right): 1743,1696,1583,1437,1250$. MS (ESI, $m / z): 435[\mathrm{M}+\mathrm{Na}]^{+}$.

Dimethyl 1,3-di-p-tolyl-1,2,3,6-tetrahydropyrimidine-4,5dicarboxylate (Table 2, Entry 3): Yield: 67\%. Color: Yellow oil. ${ }^{1} \mathrm{H}$ NMR (200 MHz, $\left.\mathrm{CDCl}_{3}, \delta, \mathrm{ppm}\right): 7.35$ (d, 2H, $J=8.0 \mathrm{~Hz}, \mathrm{Ar}-\mathrm{H}$ ), $7.22(\mathrm{~d}, 2 \mathrm{H}, J=8.0 \mathrm{~Hz}, \mathrm{Ar}-\mathrm{H}), 6.75(\mathrm{~d}, 2 \mathrm{H}, J=8.0 \mathrm{~Hz}, \mathrm{Ar}-\mathrm{H}), 6.65$ (d, $2 \mathrm{H}, J=8.0 \mathrm{~Hz}, \mathrm{Ar}-\mathrm{H}), 4.85\left(\mathrm{~s}, 2 \mathrm{H}, \mathrm{CH}_{2}\right), 4.22\left(\mathrm{~s}, 2 \mathrm{H}, \mathrm{CH}_{2}\right), 3.79$ $\left(\mathrm{s}, 3 \mathrm{H}, \mathrm{CH}_{3}\right), 3.58\left(\mathrm{~s}, 3 \mathrm{H}, \mathrm{CH}_{3}\right), 2.35,\left(\mathrm{~s}, 3 \mathrm{H}, \mathrm{CH}_{3}\right) 2.25(\mathrm{~s}, 3 \mathrm{H}$, $\left.\mathrm{CH}_{3}\right)$. FT-IR (KBr, v, cm${ }^{-1}$ ): 1746, 1697, 1584, 1438, 1255. MS (ESI, $m / z): 381[\mathrm{M}+\mathrm{H}]^{+}$.

Dimethyl 1,3-bis(4-(trifluoromethyl)phenyl)-1,2,3,6-tetra hydropyrimidine-4,5 dicarboxylate (Table 2, Entry 4): Yield: 70\%. Color: Yellow oil. ${ }^{1} \mathrm{H}$ NMR (200 $\left.\mathrm{MHz}, \mathrm{CDCl}_{3}, \delta, \mathrm{ppm}\right): 7.60$ (d, 2H, $J=8.0 \mathrm{~Hz}, \mathrm{Ar}-\mathrm{H}), 7.39$ (d, 2H, $J=8.0 \mathrm{~Hz}, \mathrm{Ar}-\mathrm{H}), 7.12$ (d, $2 \mathrm{H}, J=8.0 \mathrm{~Hz}, \mathrm{Ar}-\mathrm{H}), 6.83$ (d, 2H, $J=8.0 \mathrm{~Hz}, \mathrm{Ar}-\mathrm{H}), 4.90(\mathrm{~s}, 2 \mathrm{H}$, $\left.\mathrm{CH}_{2}\right), 4.32\left(\mathrm{~s}, 2 \mathrm{H}, \mathrm{CH}_{2}\right), 3.76\left(\mathrm{~s}, 3 \mathrm{H}, \mathrm{CH}_{3}\right), 3.63\left(\mathrm{~s}, 3 \mathrm{H}, \mathrm{CH}_{3}\right)$. FT-IR $\left(\mathrm{KBr}, v, \mathrm{~cm}^{-1}\right): 1745,1710,1609,1440$. MS (ESI, $\left.m / z\right): 489$ $[\mathrm{M}+\mathrm{H}]^{+}$.

Diethyl 1,3-bis(4-(trifluoromethyl)phenyl)-1,2,3,6-tetrahydro pyrimidine-4,5-dicarboxylate (Table 2, Entry 5): Yield: $70 \%$. Color: Yellow oil. ${ }^{1} \mathrm{H}$ NMR $\left(200 \mathrm{MHz} \mathrm{CDCl}_{3}, \delta\right.$, ppm): $7.57(\mathrm{~d}, 2 \mathrm{H}$, $\mathrm{I}=8.0 \mathrm{~Hz}, \mathrm{Ar}-\mathrm{H}), 7.42(\mathrm{~d}, 2 \mathrm{H}, J=8.0 \mathrm{~Hz}, \mathrm{Ar}-\mathrm{H}), 7.15(\mathrm{~d}, 2 \mathrm{H}, J=$ $8.0 \mathrm{~Hz}, \mathrm{Ar}-\mathrm{H}$ ), 6.79 (d, 2H, $J=8.0 \mathrm{~Hz}, \mathrm{Ar}-\mathrm{H}), 4.90\left(\mathrm{~s}, 2 \mathrm{H}, \mathrm{CH}_{2}\right.$ ), $4.33\left(\mathrm{~s}, 2 \mathrm{H}, \mathrm{CH}_{2}\right), 4.25\left(\mathrm{q}, 2 \mathrm{H}, J=7.0 \mathrm{~Hz}, \mathrm{OCH}_{2}\right), 4.10(\mathrm{q}, 2 \mathrm{H}, J=$ $7.0 \mathrm{~Hz}, \mathrm{OCH}_{2}$ ), 1.33 (t, 3H, J=7.0 Hz, CH 3$), 1.09$ (t, 3H, J=7.0 Hz, $\mathrm{CH}_{3}$ ). FT-IR $\left(\mathrm{KBr}, \mathrm{v}, \mathrm{cm}^{-1}\right): 1742,1711,1615,1330 . \mathrm{MS}$ (ESI, $m / z): 517[\mathrm{M}+\mathrm{H}]^{+}$.

\section{Results and discussion}

Cyclodextrins are cyclic oligosaccharides possessing hydrophobic cavities, which bind substrates selectively and catalyze different chemical reactions by supramolecular catalysis involving reversible formation of host-guest complexes. We describe herein, the aqueous phase synthesis of poly-substituted tetra hydro pyrimidine/pyrrolidine derivatives demonstrating the remarkable catalytic activity of $\beta$ cyclodextrin (Schemes 1 and 2). In general, the reaction was carried out by the in situ formation of the $\beta$-CD complex of aniline in water followed by the addition of but-2-ynedioate and formaldehyde. These reaction mixtures were stirred at $60{ }^{\circ} \mathrm{C}$ to give the corresponding poly-substituted tetrahydro pyrimidines/pyrrolidines at high yields (65-75\%). The reaction goes to the completion in $4.5 \mathrm{~h}$. These reactions also take place with $\alpha$-CD and $\gamma$-CD, but with lesser yields. However, $\beta$-CD was selected as the mediator since it is inexpensive and easily accessible. Several examples illustrating this simple and practical methodology are summarized in (Table 1 and 2). No byproduct formation was observed. All the compounds were characterized by ${ }^{1} \mathrm{H}$ NMR, IR, and mass spectrometry. The catalytic activity of cyclodextrins in these reactions was established by the fact that no reaction was observed in the absence of cyclodextrin. Evidence for complexation between the amine and cyclodextrin is supported by ${ }^{1} \mathrm{H}$ NMR spectroscopy. The complexation with $\beta$-CD increases the reactivity of amino group of aniline due to the intermolecular hydrogen bonding with the CD-hydroxyl groups facilitating the addition of but-2-ynedioates. Here, $\beta$-CD not only forms the inclusion complex with aniline but is also involved in the intermolecular hydrogen bonding with the guest to promote the reaction. After the completion of reaction, the reaction mixture was cooled to room temperature and $\beta-C D$ was filtered and washed with icecold water and dried. The recovered $\beta$-CD was further used with the same substrates as a catalyst and checked for the yields and catalytic activity of recovered catalyst.

\section{Conclusion}

In conclusion, a neutral aqueous phase synthesis of polysubstituted pyrimidine/pyrrolidine derivatives was developed by the reaction of the corresponding aniline with but-2ynedioate and formaldehyde promoted by $\beta$-cyclo-dextrin. These cyclodextrin-mediated aqueous phase reactions as environmentally benign methodology may find widespread application in organic and medicinal chemistry.

\section{Acknowledgement}

We thank Council of Scientific and Industrial Research, New Delhi, India, for fellowship to Jilla Shankar, Gaddam Satish and University Grants Commission for fellowship to Katla Ramesh. 


\section{References}

[1]. Sondhi, S. M.; Jain, S.; Dinodia, M.; Shuklab, R.; Raghubir, R. Bioorg. Med. Chem. 2007, 15, 3334-3344.

[2]. Srivastav, N. C.; Manning, T. D.; Kunimoto, Y.; Kumar, R. Bioorg. Med. Chem. 2007, 15, 2045-2053.

[3]. Gazivoda, T.; Plevnik, M.; Plavec, J.; Kraljevic, S.; Kralj, M.; Pavelic, K.; Balzarini, J.; Clercq, E. D.; Mintasa, M.; Silvana, R. Bioorg. Med. Chem. 2005, 13, 131-139.

[4]. Tozkoparan, B.; Yarim, M.; Sarac, S.; Ertan, M.; Kelicen, P.; Altinok, G.; Demirdamar, R. Arch. Pharm. 2000, 333, 415-420.

[5]. DeLucca, G. V.; Liang, J.; De Lucca, I. J. Med. Chem. 1999, 42, 135-152.

[6]. Dunbar, P. G.; Durant, G. J.; Rho, T.; Ojo, B.; Huzl, J. J.; Smith, D. A.; A ElAssadi, M.; Sbeih, S.; Ngur, D. O.; Periyasamy, S.; Hoss, W.; Messer, W. S. J. Med. Chem. 1994, 37, 2774-2782.

[7]. Muravyova, E. A.; Desenko, S. M.; Musatov, V. I.; Knyazeva, I. V.; Shishkina, S. V.; Shishkin, O. V.; Chebanov, V. A. J. Comb. Chem. 2007, 9, 797-803.

[8]. Swinbourne, J. F.; Hunt, H. J.; Klinkert, G. Adv. Heterocycl. Chem. 1987, 23, 103-170.

[9]. Hermecz, I.; Vasvari-Debreczy, L.; Matyus, P. In Comprehensive Heterocyclic Chemistry; Katritzky, A. R.;Rees, C. W.; Scriven, E. V. F. Eds.; Pergamon: London, 1996; Chapter 8.23, pp. 563-595.

[10]. Nair, A. C.; Jayatilleke, P.; Wang, X.; Miertus, S.; Welsh, W. J. Med. Chem. 2002, 45, 973-982.

[11]. Zamri, A.; Sirockin, F.; Abdallah, M. A. Tetrahedron 1999, 55, 51575170.

[12]. Shutalev, A. D.; Fesenko, A. A.; Cheshkov, D. A.; Goliguzov, D. V. Tetrahedron Lett. 2008, 49, 4099-4101.

[13]. Mohrle, H.; Reinhardt, H. W. Arch. Pharm. 1981, 314, 767-776.

[14]. Cho, H.; Shima, K.; Hayashimatsu, M.; Ohnaka, Y.; Mizuno, A.; Takeuchi, Y.J. Org. Chem. 1985, 50, 4227-4230.

[15]. Zhang, M.; Jiang, H. -F. J.; Liu, H. L.; Zhu, Q. H. Org. Lett. 2007, 9, 41114113.

[16]. Chanda, K.; Dutta, Ch. M.; Karim, E.; Vishwakarma J. N. J. Heterocyclic.Chem. 2004, 41, 627-631.

[17]. Das, B.; Shinde, D. B.; ShashiKanth, B.; Satyalakshmi, G. Synthesis. 2010, 16, 2823-2825.

[18]. Zhu, Q.; Jiang, H.; Li, J.; Zhang, M.; Wang, X.; Qi, C. Tetrahedron 2009 , $65,4604-4613$.

[19]. Shankar, J.; Karnakar, K.; Srinivas, B.; Nageswar, Y. V. D. Tetrahedron Lett. 2010, 51, 3938-3939.

[20]. Ramesh, K.; Murthy, S. N.; Nageswar, Y. V. D. Tetrahedron Lett. 2011, $52,2362-2366$.

[21]. Ramesh, K.; Murthy, S. N.; Karnakar, K.; Nageswar, Y. V. D. Tetrahedron Lett. 2011, 52, 3937-3941.

[22]. Ramesh, K.; Murthy, S. N.; Karnakar, K.; Nageswar, Y. V. D. Tetrahedron Lett. 2011, 52, 4734-4737.

[23]. Ramesh, K.; Murthy, S. N.; Karnakar, K.; Nageswar, Y. V. D.; Vijayalakhshmi, K.; Prabhavathi Devi, B. L. A.; Prasad, R. B. N. Tetrahedron Lett. 2012, 44, 1126-1129.

[24]. Ramesh, K.; Karnakar, K.; Satish, G.; Reddy, K. H. V.; Nageswar, Y. V. D. Tetrahedron Lett. 2012, 53, 6095-6099.

[25]. Ramesh, K.; Karnakar, K.; Satish, G.; Anil Kumar, B. S. P.; Nageswar, Y. V. D. Tetrahedron Lett. 2012, 53, 6936-6939.

[26]. Ramesh, K.; Karnakar, K.; Satish, G.; Nageswar, Y. V. D. Chin. Chem. Lett. 2012, 23, 1331-1334.

[27]. Ramesh, K.; Narayana Murthy, S.; Karnakar, K.; Nageswar, Y. V. D. Eur. J. Chem. 2012, 3(1), 119-124. 